\title{
Research on Multi-objective Optimization of Substation Project Management Based on Resource Constraint
}

\author{
Xichu Zhou ${ }^{1}$, Li Sun ${ }^{1}$, Sixia Fan ${ }^{1}$ and Bin $\mathrm{Wu}^{1, *}$ \\ ${ }^{1}$ School of Business, Shanghai Dianji University, Shanghai, China
}

\begin{abstract}
With the rapid development of China's economy, the electricity load continues to increase, the national demand for power engineering projects is also increasing.In the context of the gradual improvement of national requirements for substation projects, decision makers no longer only consider the optimal standards of project duration, cost and quality, but also the resources consumed in the construction and the degree of impact on the environment are important criteria for judging projects.However, in order to be environment-friendly and based on resource constraints, the project management of substation projects is becoming more and more complicated.In this paper, resource factors are added on the basis of the three classical indexes, and the optimal resource allocation of the project is realized under the condition of minimizing the adverse impact on the environment. The feasibility scheme of multi-objective optimization is obtained through particle swarm optimization algorithm.
\end{abstract}

\section{Introduction}

Since the 20th century, the electric power industry, as an important energy industry in China, has developed rapidly. With the increasing proportion of national investment in electric power projects, the number and scale of various electric power projects have been improved. Substation project is one of them, based on this, in recent years, the national requirements of substation project gradually improve. Many managers began to consider that the level of project management and the use of multi-objective technology can effectively improve the indicators of the project.

For multi-objective optimization problems, early scholars began to study from two quantities: time limit and cost. Siemens[1] established the optimization model of construction period and cost based on network diagram. Ei-Rayes and Kandil[2] introduced quality objectives into the traditional duration-cost optimization model and proposed the discrete optimization model of durationcost-quality of engineering projects. Bruce and Matthew[3] incorporated quality management objectives into project objective optimization, extended the standard discrete duration-cost optimization model, developed a mixed integer linear programming model, and illustrated the comprehensive optimization among duration, cost and quality through examples. Liu Yuding [4] proposed a duration-cost optimization model with cost present value and a duration-quality optimization model based on system reliability. Based on the project duration, Zhang Xiao[5] established the substation model of duration, cost, quality and environment, but the sub-environment model is too simple.Qiu Xinyun [6]added system reliability and safety factors into the quality model to build a multiobjective optimization model.

This paper takes the construction period as the independent variable, quantifies the cost and quality model, combines various uncertainties in the engineering practice, quantifies the environmental impact, and constructs the environmental model related to the construction period with deterioration factors. At the same time, considering the resource constraints of the project, the optimal resource allocation of the project on the critical path of network graph is realized on the basis of limited human resources. It provides a new idea for multiobjective optimization of substation project management.

\section{Assumptions of the model}

\subsection{Quantification of duration model}

The duration of the project construction depends on the path in the network planning diagram. In all the paths in the network planning diagram, the sum of each procedure is compared with each other. The path with the maximum value is the critical path of the project construction, and the duration calculated on the critical path is the total construction period of the project. Based on this, this paper establishes the following project duration model:

$$
\min T=\sum_{i \in A}^{n} P_{i}
$$

${ }^{*}$ Corresponding author: wub@sdju.edu.cn 


\subsection{Quantification of cost model}

Engineering cost is divided into direct cost and indirect cost. This paper quotes Zhang Xiao's multi-objective optimization model of substation project[5]:

$$
C_{i}=\sum_{i \in A}^{n}\left[b_{i}+\gamma_{i}\left(P_{n_{i}}-P_{i}\right)^{2}\right]+\alpha(L)+\beta(K)
$$

\subsection{Quantification of quality model}

The total quality of an engineering project is determined jointly by the quality of various work activities. This paper quotes Zhang Xiao's multi-objective optimization model of substation project[5]:

$$
\max Q=\sum_{i \in A}^{n} \omega_{i}^{*} \ln \left(\phi_{i} P_{i}+\varphi_{i}\right)
$$

\section{Model building}

In recent years, resource conservation and environmental protection have been the themes of national advocacy. In the construction of a project, considering the impact on environmental protection, enterprises and investors will adjust the allocation of resources according to the nature of the project in a planned way. After reviewing the relevant literature, this paper finds that the environment and other factors in the project have significant influence, and the influence amplitude is different. In this paper, after obtaining the network diagram of the project according to the critical path method, aiming at the duration of the sub-project on the critical path, the mathematical model of environmental impact is established:

$$
\min E=(d \times s) \sum_{j=1}^{m} P_{j} e_{j}+R
$$

Where, $E$ represents the proportion coefficient of the impact of the total project on the environment; $d$ represents the distance level between the construction scope and the residential gathering area; $s$ represents the noise pollution standard of construction; $e_{j}$ represents the pollution index generated by the sub-project to the surrounding environment; $R$ represents optimal resource allocation.

$$
\begin{gathered}
d=\left\{\begin{array}{l}
1+\left(1-\frac{d_{k}}{10}\right)^{2}\left(d_{k} \leq 10\right) \\
1 \quad\left(d_{k} \geq 10\right)
\end{array}\right. \\
s=\sqrt{\frac{1}{T_{0}} \int_{0}^{24}\left|80-P t_{0}\right| d t} \\
R=\sum_{j=1}^{m} u_{j} \\
u_{j}=P_{j}\left(\frac{k_{j}}{H_{j}}\right)^{\eta}
\end{gathered}
$$

$u_{j}$ represents the energy and resource consumption levels of sub-projects on the critical path; $k_{j}$ represents the assigned position on the path of the subproject; $H_{j}$ represents the allocated human resources of the subproject; $\eta$ is a deterioration factor.

In general engineering, in order to improve the efficiency of the project, the maximum human resource factor of the project will be selected in advance in the stage of drawing up the plan.Therefore:

$$
\sum_{j \in B}^{m} H_{j} \leq G
$$

Where, $G$ represents the maximum human resource ratio coefficient, and $B$ represents the set of sub-projects on the critical path.

And since $E$ is only dependent on $R$ and $H_{j}$, and $(d \times s) \sum_{j=1}^{m} P_{j} e_{j}$ is independent of $H_{j}$, under the constraints of $\sum_{j \in B}^{m} H_{j} \leq G$, you can view $P_{j}$ it as a constant quantity, so if you minimize $E$, you're minimizing $R$.So based on this constraint, the objective function is:

$$
\min R=\sum_{j \in B}^{m} u_{j}=\sum_{j \in B}^{m} P_{j}\left(\frac{k_{j}}{H_{j}}\right)^{\eta}
$$

Obviously, the target function $R$ is minimized under $\sum_{j \in B}^{m} H_{j}=G$.Therefore, the problem becomes a conditional extremum problem when the constraint conditions are satisfied.To solve this problem, we adopt the Lagrange Multiplier Method, whose function is

$$
L(H, \lambda)=\sum_{j=1}^{m} P_{[j]}\left(\frac{k_{[j]}}{H_{[j]}}\right)^{\eta}+\lambda\left(\sum_{j=1}^{m} H_{[j]}-G\right)
$$

Since the objective function is convex, take the partial derivatives of $H_{[j]}$ and $\lambda$, respectively, in Formula (11), and set the derivative function as 0 , so we can get:

$$
\begin{gathered}
\frac{\partial L(H, \lambda)}{\partial H_{[j]}}=\lambda-\eta P_{[j]} \frac{k_{[j]}^{\eta}}{H_{[j]}^{\eta+1}}=0 \\
\frac{\partial L(H, \lambda)}{\partial \lambda}=\sum_{j=1}^{m} H_{[j]}-G=0
\end{gathered}
$$

According to Formula (12), we can get:

$$
H_{[j]}^{*}=\eta^{\frac{1}{\eta+1}} P_{[j]}{ }^{\frac{1}{\eta+1}} \frac{k_{[j]}^{\frac{\eta}{\eta+1}}}{\lambda^{\frac{1}{\eta+1}}}
$$

And according to Formula(13) and Formula(14),we can get : 


$$
\lambda^{\frac{1}{\eta+1}}=\mathrm{G}^{-1} \eta^{\frac{1}{\eta+1}} \sum_{j=1}^{m} P_{[j]}^{\frac{1}{\eta+1}} k_{[j]}^{\frac{\eta}{\eta+1}}
$$

Substituting Equation (15) into Equation (14) can be obtained :

$$
H_{[j]}=\frac{G P_{[j]}^{\frac{1}{\eta+1}} k_{[j]}^{\frac{\eta}{\eta+1}}}{\sum_{j=1}^{m} P_{[j]}^{\frac{1}{\eta+1}} k_{[j]}^{\frac{\eta}{\eta+1}}}
$$

Based on the above conditions, update the energy and resource consumption level:

$$
u_{j}=G^{-\eta} P_{j}^{\frac{1}{\eta+1}} k_{j}^{\frac{\eta}{\eta+1}}\left(\sum_{j=1}^{m} P_{j}^{\frac{1}{\eta+1}} k_{j}^{\frac{\eta}{\eta+1}}\right)^{\eta}
$$

So in this case, the final amount of resources is

$$
\begin{aligned}
R= & G^{-\eta}\left(\sum_{j=1}^{m} P_{j}^{\frac{1}{\eta+1}} k_{j}{ }^{\frac{\eta}{\eta+1}}\right)^{\eta+1} \\
& \left\{\begin{array}{l}
\tau_{j} \leq P_{j} \leq \mu_{j} \\
k_{j} \in B \\
j=1,2, \cdots m
\end{array}\right.
\end{aligned}
$$

Based on the hypothesis of the model, it can be seen that cost, duration and quality are the main influencing factors of the project. Combined with the above theoretical derivation and adding the environmental objective function, the following four-objective optimization model of project duration, cost, quality and environment can be established:

$$
\left\{\begin{array}{l}
\min T=\sum_{j=1}^{m} P_{j} \\
\min C=\sum_{i \in A}^{n}\left[b_{i}+\gamma_{i}\left(n_{i}-P_{i}\right)^{2}\right]+\alpha(L)+\beta(K) \\
\max Q=\sum_{i \in A}^{n} \omega_{i}^{*} \ln \left(\phi_{i} P_{i}+\varphi_{i}\right) \\
\min E=(d \times s) \sum_{j=1}^{m} P_{j} e_{j}+R
\end{array}\right.
$$

$$
\begin{aligned}
& R=G^{-\eta}\left(\sum_{j=1}^{m} P_{j}^{\frac{1}{\eta+1}} k_{j}^{\frac{\eta}{\eta+1}}\right)^{\eta+1}, \phi_{i}=\frac{e-e^{q i}}{D m_{i}-D c_{i}}, \\
& \varphi_{i}=\frac{e^{q i} * D m_{i}-e^{*} D c_{i}}{D m_{i}-D c_{i}}, L=T_{\text {stipulate }}-\sum P_{i} \text {, } \\
& K=\sum P_{i}-T_{\text {stipulate }}
\end{aligned}
$$

$$
\left\{\begin{array}{l}
\tau_{i} \leq P_{i} \leq \mu_{i}, i \in A \\
\min T \leq T_{d} \\
\omega_{i}>0 \\
\sum_{i \in A} \omega_{i}=1 \\
0 \leq Q_{i} \leq 1 \\
\sum \omega_{i}^{*} \ln \left(\phi_{i} P_{i}+\varphi_{i}\right) \geq Q_{d}
\end{array}\right.
$$

\section{The empirical research}

The construction period of A substation electric power engineering project is 195 245 days, and the construction period required by the contract is 225 days, and the indirect cost is 3000 yuan/day. The quality level is excellent, equal to the quality level of not less than 0.9 . If the construction is completed one day ahead of schedule, 4,000 yuan will be awarded, and each day delayed, 5,000 yuan will be fined. After all kinds of data analysis, data collection, induction and summary of the project as well as preliminary data processing. Draw the network diagram of the project and the relevant parameter table as follows:

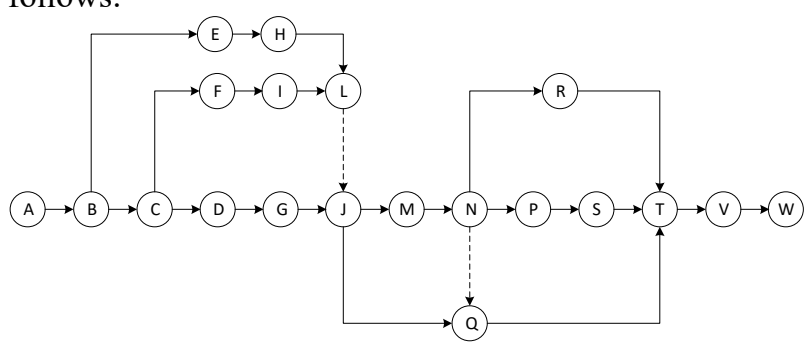

Fig. 1. Project network diagram.

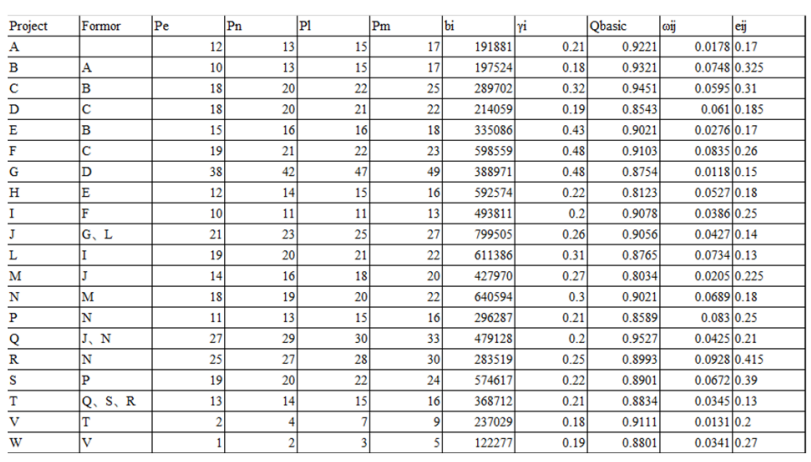

Fig. 2. Engineering data.

After expert evaluation and inspection, the construction site of the project is about $7.6 \mathrm{~km}$ away from the residential gathering area. Therefore

$$
d=1+\left(1-\frac{7.6}{10}\right)^{2}=1.0576
$$

The average noise $\mathrm{Pt}$ in a day is $60 \mathrm{~dB}$, so

$$
s=\sqrt{\frac{1}{225} \int_{0}^{24}|80-60| d t}=1.46
$$

The critical path of solving network graph using network planning technology is as follows: 


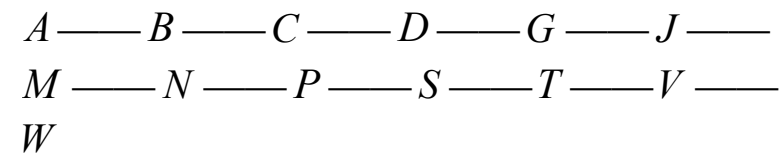

In Matlab2020A, the program of multi-objective particle swarm optimization algorithm can obtain 50 Pareto optimal solutions after one run, and 50 Pareto optimal solution sets can be obtained after about 500 iterations of the calculation process. The convergence process is shown in the figure:

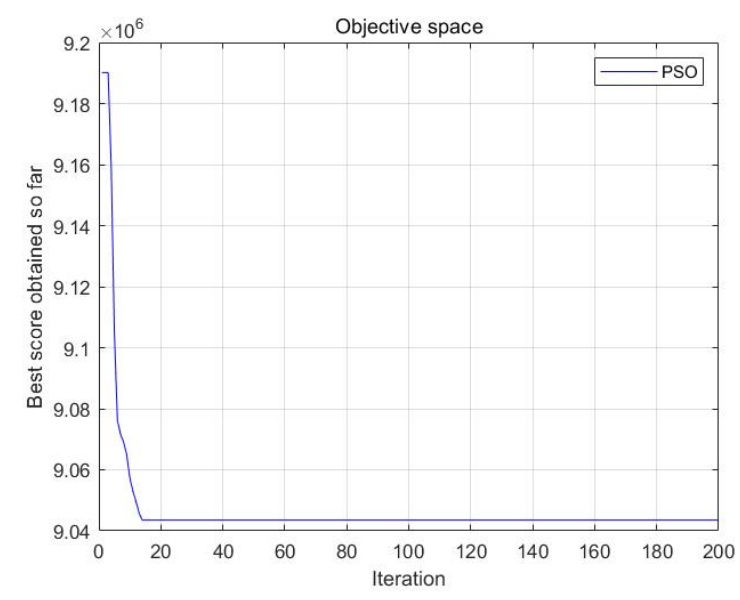

Fig. 3. Convergent line chart.

Due to the real implementation of the project, the actual design is often expected to be different, in the actual project, the construction period, cost, quality and environment for the final level of construction focus is also different. Managers may prefer the optimal cost, while construction workers prefer the shortest construction period. Based on the different emphasis of each optimization quantity, we select four groups of optimal result points relative to each index from the 50 solution sets (as shown in the table below):

Table 1. 4 optimal solutions of Paroto.

\begin{tabular}{|c|c|c|c|c|}
\hline $\begin{array}{c}\text { Pareto } \\
\text { optimal } \\
\text { solutions }\end{array}$ & $\mathrm{T}$ & $\mathrm{C}$ & $\mathrm{Q}$ & $\mathrm{E}$ \\
\hline 1 & 198 & $\begin{array}{r}907040 \\
9.0775\end{array}$ & 0.9475 & 77.1973 \\
\hline 2 & 196 & $\begin{array}{r}912346 \\
7.7599\end{array}$ & 0.9472 & 77.3752 \\
\hline 3 & 200 & $\begin{array}{r}909720 \\
8.8569\end{array}$ & 0.9431 & 75.7002 \\
\hline 4 & 199 & $\begin{array}{r}905221 \\
0.8497\end{array}$ & 0.9403 & 76.9947 \\
\hline
\end{tabular}

It can be seen from the table that the combination of Pareto optimal solutions obtained by this method can make the decision maker's plan not simple, and can be used to solve the requirements of substation project in various objectives. Scheme 1 is more suitable for high quality projects, Scheme 2 is suitable for short construction period and fast construction, Scheme 3 is suitable for projects with minimum environmental damage and higher environmental requirements, and Scheme 4 is suitable for the pursuit of the lowest cost. The decision maker can choose the appropriate project plan according to the actual project and the requirements of the project.

\section{Conclusion}

In this paper, on the premise of the traditional substation project to consider the optimization of the three factors, added and quantified the standard of resources and environment, the application of the model established in this paper and the solution method, can effectively solve the actual project for the project duration, cost, quality and resources of the comprehensive optimization problems. Because the project is a huge and complex system engineering, there are many kinds and different specific requirements, so the optimization of the four control objectives needs to be further in-depth. This paper explores the optimal environmental proportion of the subprojects on the critical path and optimizes resource allocation. For the sub-projects on the non-critical path, resource allocation and environmental proportion need to be further studied.

\section{Acknowledgement}

Humanity and Social Science Youth foundation of Ministry of Education of China (Project No.20YJCZH027)

\section{References}

1. Nicolai Siemens. A Simple CPM Time-Cost Tradeoff Algorithm[J]. Management Science,1971,17(6).

2. Khaled El-Rayes, Amr Kandil. Time-Cost-Quality Trade-Off Analysis for Highway Construction[J]. Journal of Construction Engineering and Management, 2005, 131(4).

3. B. Pollack-Johnson and M. J. Liberatore, "Incorporating Quality Considerations Into Project Time/Cost Tradeoff Analysis and Decision Making," in IEEE Transactions on Engineering Management, vol. 53, no. 4, pp. 534-542, Nov. 2006, doi: 10.1109/TEM.2006.883705.

4. Liu Y. 2016.Research on Multi-objective Dynamic Optimization of Construction Project Based on Improved Particle Swarms Optimization.MD thesis, HUE Hebei University of Engineering.

5. Zhang X. 2018.Research on Multi-objective Optimization of Substation Project Based on Particle Swarms Optimization Algorithm.MD thesis, NCEPU North China Electric Power University.

6. Zhuo J. \& Lu H. Research on Multi-objective Optimization of Engineering Projects Based on Particle Swarms Optimization Algorithm [J]. Journal of Engineering Management,2017,31(06):101-106. 